\title{
A Systematic Review of Genetic Testing and Lifestyle Behaviour Change: Are We Using High-Quality Genetic Interventions and Considering Behaviour Change Theory?
}

\author{
Justine Horne ${ }^{\mathrm{a}, \mathrm{b}}$ Janet Madill ${ }^{\mathrm{b}}$ Colleen O'Connor ${ }^{\mathrm{b}}$ Jacob Shelley ${ }^{\mathrm{c}-\mathrm{e}}$ \\ Jason Gilliland ${ }^{\mathrm{d}, \mathrm{f}-\mathrm{h}}$

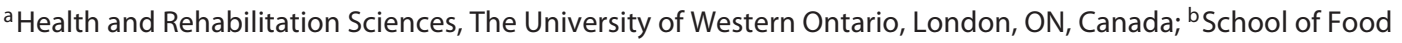 \\ and Nutritional Sciences, Brescia University College at The University of Western Ontario, London, ON, Canada; \\ ${ }^{c}$ Faculty of Law, The University of Western Ontario, London, ON, Canada; ${ }^{d}$ School of Health Studies, The University

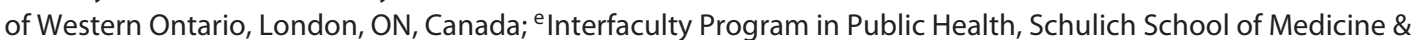 \\ Dentistry, The University of Western Ontario, London, ON, Canada; ${ }^{\mathrm{f} D e p a r t m e n t}$ of Geography, The University of \\ Western Ontario, London, ON, Canada; ' ${ }^{D}$ epartment of Paediatrics, The University of Western Ontario, London, ON, \\ Canada; ' hepartment of Epidemiology and Biostatistics, The University of Western Ontario, London, ON, Canada
}

\section{Keywords}

Health behaviour - Genetics · Nutrition - Physical activity · Smoking · Personalized healthcare $\cdot$ Nutrigenomics ·

Lifestyle genomics

\begin{abstract}
Background: Studying the impact of genetic testing interventions on lifestyle behaviour change has been a priority area of research in recent years. Substantial heterogeneity exists in the results and conclusions of this literature, which has yet to be explained using validated behaviour change theory and an assessment of the quality of genetic interventions. The theory of planned behaviour (TPB) helps to explain key contributors to behaviour change. It has been hypothesized that personalization could be added to this theory to help predict changes in health behaviours. Purpose: This systematic review provides a detailed, comprehensive identification, assessment, and summary of primary research articles pertaining to lifestyle behaviour change (nutrition,
\end{abstract}

physical activity, sleep, and smoking) resulting from genetic testing interventions. The present review further aims to provide in-depth analyses of studies conducted to date within the context of the TPB and the quality of genetic interventions provided to participants while aiming to determine whether or not genetic testing facilitates changes in lifestyle habits. This review is timely in light of a recently published "call-to-action" paper, highlighting the need to incorporate the TPB into personalized healthcare behaviour change research. Methods: Three bibliographic databases, one key website, and article reference lists were searched for relevant primary research articles. The PRISMA Flow Diagram and PRISMA Checklist were used to guide the search strategy and manuscript preparation. Out of 32,783 titles retrieved, 26 studies met the inclusion criteria. Three quality assessments were conducted and included: (1) risk of bias, (2) quality of genetic interventions, and (3) consideration of theoretical underpinnings - primarily the TPB. Results: Risk of bias in studies was overall rated to be "fair." Consideration of the TPB was "poor," with no study making reference to this

\section{KARGER}

(c) 2018 S. Karger AG, Basel

E-Mail karger@karger.com

www.karger.com/lfg
Justine Horne

Faculty of Health Sciences, The University of Western Ontario

1151 Richmond Street

London, ON N6A 3K7 (Canada)

E-Mail jhorne5@uwo.ca 
validated theory. While some studies $(n=11 ; 42 \%)$ made reference to other behaviour change theories, these theories were generally mentioned briefly, and were not thoroughly incorporated into the study design or analyses. The genetic interventions provided to participants were overall of "poor" quality. However, a separate analysis of studies using controlled intervention research methods demonstrated the use of higher-quality genetic interventions (overall rated to be "fair"). The provision of actionable recommendations informed by genetic testing was more likely to facilitate behaviour change than the provision of genetic information without actionable lifestyle recommendations. Several studies of good quality demonstrated changes in lifestyle habits arising from the provision of genetic interventions. The most promising lifestyle changes were changes in nutrition. Conclusions: It is possible to facilitate behaviour change using genetic testing as the catalyst. Future research should ensure that high-quality genetic interventions are provided to participants, and should consider validated theories such as the TPB in their study design and analyses. Further recommendations for future research are provided.

(c) 2018 S. Karger AG, Basel

\section{Introduction}

Since decoding the entire human genome in 2003 [1], there have been considerable advances in genetic research and the clinical utility of genetic testing. The terms nutrigenomics or nutritional genomics describe the study of how genes interact with the foods, beverages, and supplements consumed to influence health outcomes [2]. Currently, there are no generally accepted or standardized terms describing the study of how genes interact with physical activity, sleep, or smoking to influence subsequent health outcomes. These gene-lifestyle interactions can be referred to using the broad term lifestyle genomics. Despite the lack of a standardized terminology, research pertaining to nutrigenomics and other emerging genomic sciences continues to advance. Specifically, behaviour change guided by genetic testing results or other personalized healthcare information is emerging as a priority area of research, with several reviews on this topic published in recent years [3-6].

Genetic testing is increasingly used in clinical practice to provide personalized information and recommendations about health risks and lifestyle habits at a relatively low cost [7]. However, studies assessing whether or not genetic testing promotes changes in lifestyle habits have conflicting findings [8-11]. Given that chronic diseases can often be managed through lifestyle interventions alone, or a combination of lifestyle interventions and medication [12-14], genetic tests providing personalized lifestyle recommendations hold considerable promise.

Behaviour change is a multifactorial, complex area of research and clinical practice. The theory of planned behaviour (TPB) is arguably the most widely accepted behaviour change theory in academia [15]. This theory posits that attitudes, subjective norms, and perceived behavioural control are key constructs that can be used to predict behaviours. Actual behavioural control, which typically refers to factors such as income, educational level, and other social determinants of health for the purposes of healthcare research, further contributes to one's likelihood of performing a behaviour $[15,16]$. It is important for genetic testing behaviour change research to consider validated theories in order to control for a number of confounding factors that could significantly influence the results of a study.

Despite the complexity of behaviour change, genetic testing behaviour change studies do not often use any theoretical underpinnings to inform their study design, or for the analysis and interpretation of their data. This is concerning, as it implies that these studies did not report whether they considered the many confounding factors impacting behaviour change, including but not limited to attitudes, subjective norms, and perceived and actual behavioural control [17]. Consideration of such factors could help explain why some studies conclude that genetic testing facilitates health behaviour change, while others conclude that it does not. For example, a study may find that genetic testing has a positive influence on attitudes and subjective norms, but it is only when behavioural control is high (for example, with a higher income or education level) that genetic testing facilitates health behaviour change. The importance of such considerations has been highlighted in a recent call to action for personalized healthcare behaviour change research, which recommended the completion of a systematic review with perspective from the TPB as an important next step in advancing knowledge in personalized healthcare behaviour change literature [18].

Systematic reviews and meta-analyses are typically considered the highest quality of scientific evidence and, notably, often guide clinical practice [19]. When it comes to systematic reviews assessing behaviour change as a result of genetic testing interventions, a simple risk-of-bias assessment is not sufficient to develop the most meaningful conclusions; yet it is often the only quality assessment conducted in this type of work $[3,5,6]$. It is further im- 
Fig. 1. Search terms.

\begin{tabular}{|c|}
\hline $\begin{array}{c}\text { nutrigenetic* OR nutrigenomic* OR DNA OR "nutritional genomic*" OR gene OR genes OR } \\
\text { genetic* OR genom* OR "personalized nutrition" OR "personalised nutrition" }\end{array}$ \\
\hline AND \\
\hline behavior* OR behaviour* OR habit* OR smoking OR smoke* OR diet OR nutrition OR "physical \\
activity" OR exercise OR sleep
\end{tabular}

portant to consider the delivery of a health/genetic intervention (such as considering the provision of disease risk estimates vs. actionable behaviour change recommendations) and to consider behaviour change theories [18]. Therefore, the development of more comprehensive methods for reviewing and compiling the primary research articles conducted to date related to genetic testing behaviour change is needed.

The present review provides an in-depth analysis and summary of the current body of knowledge, thus presenting the most robust and comprehensive review of genetic testing behaviour change research conducted to date. Overall, the purpose of this comprehensive systematic review is to use these novel perspectives to answer the following research questions: Are we considering validated behaviour change theory (particularly the TPB) in genetic testing behaviour change research? Are we using highquality genetic interventions in genetic testing behaviour change research? What is the impact of genetic testing on behaviour change pertaining to four lifestyle factors: nutrition, physical activity, smoking, and/or sleep? These four lifestyle factors were chosen as they have all been shown to have a significant impact on chronic disease management [20-24]. Behaviour change is challenging, and it is important to find strategies that effectively facilitate beneficial lifestyle changes related to nutrition, physical activity, smoking, and/or sleep. Genetic tests may provide information on disease risk, which can be mitigated through specific alterations in lifestyle habits such as improving nutrition, optimizing physical activity habits, quitting smoking or smoking less, and engaging in healthful sleep-related behaviours.

\section{Methods}

\section{Search Strategy}

The systematic review protocol that was used to guide this review is detailed elsewhere [25]. In brief, the search strategy was guided by the PRISMA Flow Diagram [26]. From February to April 2017, the following databases were searched for relevant ar- ticles: PubMed, Scopus, and Nursing \& Allied Health. Publications posted on the Food4Me website [27], as well as the reference lists of 4 recent review articles published on topics similar to those of the present review [3-6], were also screened for articles relevant to the research questions. After the number of records had been condensed through title and abstract screening, the full-text articles were reviewed to assess each one for eligibility according to predetermined inclusion and exclusion criteria. The complete search terms and search strategy were developed and approved by all authors, and they are detailed in Figure 1 and Figure 2, respectively.

\section{Selection Criteria}

To capture a comprehensive summary of the research conducted to date, the present review was not limited to a single, specific study design. We included primary research articles published in English in peer-reviewed journals from all years which assessed the impact of genetic testing on one or more of the four lifestyle habits of interest (nutrition, physical activity, smoking, and/or sleep). Both qualitative and quantitative studies were included. Studies were excluded if there was not at least one group of participants who underwent genetic testing and/or if the study did not provide follow-up data related to one or more of the lifestyle habits of interest after the participants had received the results of a genetic test. One author (J.H.) completed data extraction using piloted forms [28], which were tested on 4 studies, reviewed by another author (J.G.), and modified during the piloting process by two authors (J.H. and J.G.).

\section{Analysis}

The National Institutes of Health (NIH) Study Quality Assessment Tools were used to conduct a risk-of-bias assessment in quantitative research [29]. The Critical Appraisal Skills Programme Qualitative Research Checklist [30] was used to assess risk of bias in qualitative research. The quality of the genetic intervention was also assessed. To our knowledge, there currently is no tool available for assessing the quality of a genetic intervention. As such, we developed the first assessment tool for evaluating the quality of a genetic intervention provided to subjects (online suppl. Table 1; see www.karger.com/doi/10.1159/000488086 for all online suppl. material). The quality rating and general outline for this new tool was based on the format of the NIH Study Quality Assessment Tools [29]. The questions included were developed from a review of previously identified critiques and concerns related to genetic testing and health risk messages [11, 31-38].

Consideration of the main components of the TPB (attitudes towards a behaviour, subjective norms, behavioural control, and intention) [17], as well as consideration of theory more generally, was assessed using deductive content analysis of the manuscripts 


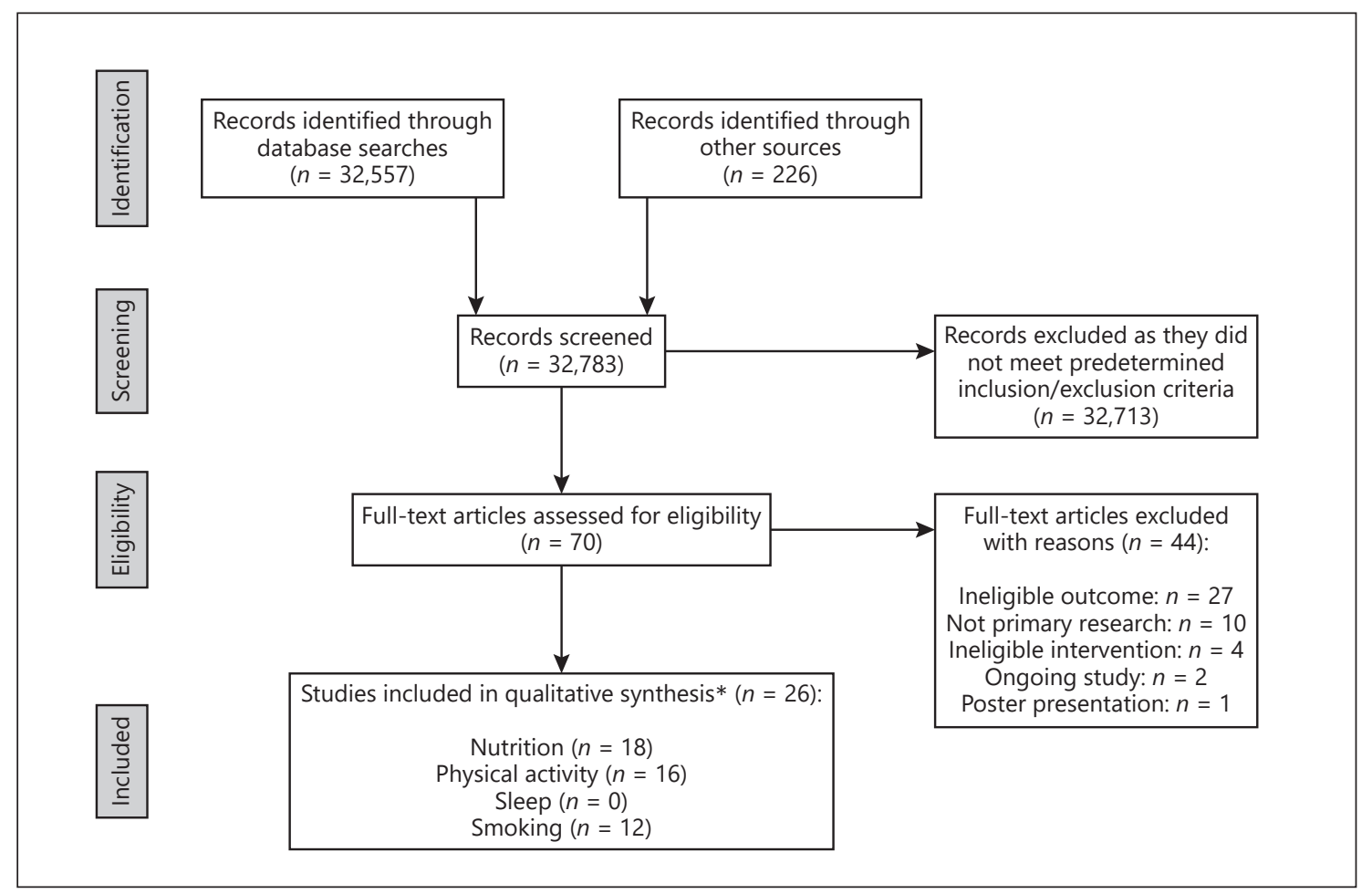

Fig. 2. Search strategy guided by the PRISMA Flow Diagram. * Several articles assessed behaviour change related to $>1$ lifestyle factor of interest; therefore, the total number of records included in the systematic review does not match the total number of articles by lifestyle category.

[39]. The deductive content analyses of consideration of the TPB and its key components in each study was then translated into a rating, based on the rating system generated in the NIH Study Quality Assessment Tools, whereby "good" indicates a robust consideration of the main components of the TPB, "fair" indicates intermediate consideration of the main TPB components, and "poor" represents little to no consideration of the main TPB components. An overall quality score was assigned to each article based on a point system, where "good" ratings were awarded 3 points, "fair" ratings were awarded 2 points, and "poor" ratings were awarded 1 point. The maximum possible overall quality rating was $9 / 9$, upon consideration of all three assessments.

\section{Results}

The comprehensive electronic literature search returned a total of 32,783 results, with 26 studies meeting the predetermined inclusion criteria. In these 26 studies, the following outcomes were assessed: nutrition $(n=18)$, physical activity $(n=16)$, and smoking $(n=12)$ (Fig. 1$)$, with 14 articles assessing more than one lifestyle habit of interest to this review. The vast majority of the literature has been published over the past decade, with a large spike in publications recently in 2015 (online suppl. Fig. 1). Consistent with recommendations for systematic reviews [25], our review was analytic and descriptive in nature and included: (a) a tabulation of the study characteristics and findings (Table 1); (b) a thorough and robust quality assessment (Table 2); and (c) a narrative synthesis. Research conducted thus far has focused on a variety of genes, as outlined in Table 3 . It is concerning to note that 12 studies (46\%) did not report whether or not the authors had a conflict of interest (COI). The vast majority of the literature has focused on genetic testing for determining the risk of developing certain diseases or conditions $(88 \% ; n=23)$, while only a small number of studies have focused on nutrient metabolism $(12 \% ; n=3)$, which indirectly affects the risk of developing diseases or conditions [40-42]. The three separate quality assessments completed on each study are summarized in Table 2. Risk of bias was overall rated as "fair."

\section{Are We Using High-Quality Genetic Interventions?}

Although some risk of bias is apparent, the ratings for the quality of the genetic interventions were more con- 
Table 1. Summary of study characteristics and behaviour change findings

\begin{tabular}{|c|c|c|c|c|c|c|c|c|c|}
\hline $\begin{array}{l}\text { First author } \\
\text { [Ref.], date }\end{array}$ & $\begin{array}{l}\text { Participants } \\
\text { ( } n \text { baseline; } \\
n \text { follow-up) }\end{array}$ & $\begin{array}{l}\text { Interven- } \\
\text { tion } \\
\text { group(s) }\end{array}$ & $\begin{array}{l}\text { Comparison } \\
\text { group }(\mathrm{s})\end{array}$ & $\begin{array}{l}\text { Target diseases/ } \\
\text { conditions } \\
\text { (genes tested) }\end{array}$ & Follow-up & $\begin{array}{l}\text { Lifestyle habits } \\
\text { assessed }\end{array}$ & $\begin{array}{l}\text { Outcomes ( } p \text { values); } \\
\text { conclusions }\end{array}$ & $\begin{array}{l}\text { Ranking } \\
\text { of study } \\
\text { design }^{1}\end{array}$ & $\mathrm{COI}$ \\
\hline $\begin{array}{l}\text { Roke [43], } \\
2017\end{array}$ & $\begin{array}{l}\text { Young female } \\
\text { adults } \\
(n=57 \\
n=56)\end{array}$ & $\begin{array}{l}\text { Genetic } \\
\text { testing }\end{array}$ & $\begin{array}{l}\text { No genetic } \\
\text { testing }\end{array}$ & $\begin{array}{l}\text { Health effects related } \\
\text { to omega- } 3 \text { intake } \\
\text { (FADS1) }\end{array}$ & 3 months & $\begin{array}{l}\text { Nutrition } \\
\text { (omega-3: } \\
\text { EPA and DHA) }\end{array}$ & $\begin{array}{l}\text { NS change in omega-3 } \\
\text { intake in the genetic } \\
\text { testing group compared } \\
\text { to the control group (no } \\
\text { genetic testing) }\end{array}$ & 1 & No \\
\hline $\begin{array}{l}\text { Marsaux [10], } \\
2016\end{array}$ & $\begin{array}{l}\text { Adults } \\
(n=265 \\
n=130)\end{array}$ & $\begin{array}{l}\text { High-risk } \\
\text { genetic } \\
\text { result }\end{array}$ & $\begin{array}{l}\text { Non-risk } \\
\text { genetic result }\end{array}$ & $\begin{array}{l}\text { Overweight/ } \\
\text { obesity (FTO) }\end{array}$ & 6 months & Physical activity & $\begin{array}{l}\text { NS change in subjective } \\
\text { or objective physical } \\
\text { activity with provision of } \\
\text { FTO genotype risk info }\end{array}$ & 3 & Yes \\
\hline $\begin{array}{l}\text { Meisel [44], } \\
2015\end{array}$ & $\begin{array}{l}\text { Young adults } \\
(n=1,016 \\
n=279)\end{array}$ & $\begin{array}{l}\text { Genetic } \\
\text { testing }\end{array}$ & $\begin{array}{l}\text { No genetic } \\
\text { testing }\end{array}$ & Obesity (FTO) & 1 month & $\begin{array}{l}\text { Nutrition (adher- } \\
\text { ence to a variety } \\
\text { of eating behav- } \\
\text { iours) and physi- } \\
\text { cal activity }\end{array}$ & $\begin{array}{l}\text { NS changes in nutrition } \\
\text { and physical activity } \\
\text { (pooled) between groups }\end{array}$ & 1 & No \\
\hline $\begin{array}{l}\text { Boeldt [45], } \\
2015\end{array}$ & $\begin{array}{l}\text { Adults working } \\
\text { at health and } \\
\text { technology } \\
\text { companies } \\
(\mathrm{NR} ; n=2,037)\end{array}$ & $\begin{array}{l}\text { Genetic } \\
\text { testing }\end{array}$ & None & $\begin{array}{l}23 \text { conditions includ- } \\
\text { ing heart attack, } \\
\text { Alzheimer disease, } \\
\text { type } 2 \text { diabetes, } \\
\text { obesity, colon cancer, } \\
\text { and cervical cancer } \\
\text { (NR) }\end{array}$ & $\begin{array}{l}5.6 \pm 2.4 \\
\text { months }\end{array}$ & $\begin{array}{l}\text { Nutrition } \\
\text { (dietary fat) and } \\
\text { physical activity }\end{array}$ & $\begin{array}{l}\text { NS (significance level } \\
\text { NR) change in nutrition } \\
\text { and physical activity } \\
\text { following genetic testing }\end{array}$ & 4 & No \\
\hline $\begin{array}{l}\text { Hieteranta- } \\
\text { Luoma [9], } \\
2015\end{array}$ & $\begin{array}{l}\text { Adults } \\
(n=122 ; \\
n=113 \text { at } \\
12 \text { months })\end{array}$ & $\begin{array}{l}\text { Genetic } \\
\text { testing } \\
\text { and } \\
\text { High-risk } \\
\text { genetic } \\
\text { result }\end{array}$ & $\begin{array}{l}\text { No genetic } \\
\text { testing } \\
\text { and } \\
\text { Non-risk } \\
\text { genetic result }\end{array}$ & $\begin{array}{l}\text { Cardiovascular } \\
\text { disease (apoE) }\end{array}$ & $\begin{array}{l}2 \text { weeks } \\
6 \text { months } \\
12 \text { months }\end{array}$ & $\begin{array}{l}\text { Nutrition (fat } \\
\text { quality, and } \\
\text { consumption of } \\
\text { vegetables, ber- } \\
\text { ries, fruits, and } \\
\text { fatty and sugary } \\
\text { foods) and physi- } \\
\text { cal activity }\end{array}$ & $\begin{array}{l}\text { Improved dietary fat } \\
\text { quality in the high-risk } \\
\text { genetic result group vs. } \\
\text { the control group at } 2 \\
\text { weeks }(p<0.05) \text { and } 6 \\
\text { months of follow-up ( } p< \\
0.05) \text {; decreased intake of } \\
\text { high-fat, high-sugar } \\
\text { foods in the non-risk } \\
\text { genetic result group vs. } \\
\text { the control group at } 12 \\
\text { months }(p<0.05)\end{array}$ & 1 & No \\
\hline $\begin{array}{l}\text { Voils [46], } \\
2015\end{array}$ & $\begin{array}{l}\text { Veterans } \\
(n=601 ; \\
n=506 \text { at } \\
3 \text { months, } \\
n=472 \text { at } \\
6 \text { months })\end{array}$ & $\begin{array}{l}\text { Genetic } \\
\text { testing }\end{array}$ & $\begin{array}{l}\text { No genetic } \\
\text { testing }\end{array}$ & $\begin{array}{l}\text { Type } 2 \text { diabetes } \\
\text { (TCF7L2, PPAR } \gamma \text {, } \\
\text { and KCNJ11) }\end{array}$ & $\begin{array}{l}3 \text { months } \\
6 \text { months }\end{array}$ & $\begin{array}{l}\text { Nutrition (calo- } \\
\text { ries, carbohy- } \\
\text { drates, protein, } \\
\text { fat, saturated fat, } \\
\text { MUFA, and } \\
\text { PUFA) and } \\
\text { physical activity }\end{array}$ & $\begin{array}{l}\text { Reduced calories and fat } \\
\text { (MUFA and PUFA) in } \\
\text { the genetic testing group } \\
\text { vs. the no-genetic-testing } \\
\text { group ( } p<0.05 \text { ) at } 3 \\
\text { months; NS changes in } \\
\text { nutrition between the } \\
\text { groups at } 6 \text { months; NS } \\
\text { changes in physical } \\
\text { activity at either time } \\
\text { point }\end{array}$ & 1 & Yes \\
\hline $\begin{array}{l}\text { Marsaux [47], } \\
2015\end{array}$ & $\begin{array}{l}\text { Adults } \\
(n=1,607 ; \\
n=1,233 \text { with } \\
\text { subjective data } \\
\text { at } 6 \text { months, } \\
n=730 \text { with ob- } \\
\text { jective data at } \\
6 \text { months) }\end{array}$ & $\begin{array}{l}\text { Genetic } \\
\text { testing }\end{array}$ & $\begin{array}{l}\text { No genetic } \\
\text { testing }\end{array}$ & $\begin{array}{l}\text { Overweight/ } \\
\text { obesity (FTO) }\end{array}$ & $\begin{array}{l}3 \text { months } \\
6 \text { months }\end{array}$ & Physical activity & $\begin{array}{l}\text { NS changes in physical } \\
\text { activity with the addition } \\
\text { of genetic information }\end{array}$ & 1 & Yes \\
\hline $\begin{array}{l}\text { Nielsen [11], } \\
2014\end{array}$ & $\begin{array}{l}\text { Adults } \\
(n=138 ; \\
n=130 \text { at } \\
3 \text { months, } \\
n=123 \text { at } \\
12 \text { months })\end{array}$ & 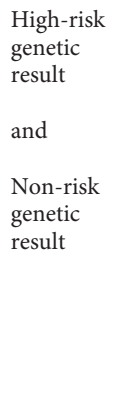 & $\begin{array}{l}\text { No genetic } \\
\text { testing }\end{array}$ & $\begin{array}{l}\text { Caffeine metabolism } \\
\text { (CYP1A2), vitamin C } \\
\text { utilization (GSTT1 } \\
\text { and GSTM1), sweet } \\
\text { taste perception } \\
\text { (TAS1R2), and } \\
\text { sodium sensitivity } \\
\text { (ACE) }\end{array}$ & $\begin{array}{l}3 \text { months } \\
12 \text { months }\end{array}$ & $\begin{array}{l}\text { Nutrition (caf- } \\
\text { feine, vitamin C, } \\
\text { added sugar, and } \\
\text { sodium) }\end{array}$ & $\begin{array}{l}\text { The high-risk genetic } \\
\text { result group (for the } \\
\text { ACE gene) had reduced } \\
\text { sodium intake to a } \\
\text { greater extent than the } \\
\text { control group by the } \\
\text { 12-month follow-up ( } p= \\
0.008 \text { ); NS changes in } \\
\text { caffeine, vitamin C, and } \\
\text { added sugar intake at } \\
\text { each follow-up time } \\
\text { point; NS changes in } \\
\text { sodium intake at the } \\
\text { 3-month follow-up }\end{array}$ & 1 & Yes \\
\hline
\end{tabular}


Table 1 (continued)

\begin{tabular}{|c|c|c|c|c|c|c|c|c|c|}
\hline $\begin{array}{l}\text { First author } \\
\text { [Ref.], date }\end{array}$ & $\begin{array}{l}\text { Participants } \\
\text { ( } n \text { baseline; } \\
n \text { follow-up) }\end{array}$ & $\begin{array}{l}\text { Interven- } \\
\text { tion } \\
\text { group(s) }\end{array}$ & $\begin{array}{l}\text { Comparison } \\
\text { group }(s)\end{array}$ & $\begin{array}{l}\text { Target diseases/ } \\
\text { conditions } \\
\text { (genes tested) }\end{array}$ & Follow-up & $\begin{array}{l}\text { Lifestyle habits } \\
\text { assessed }\end{array}$ & $\begin{array}{l}\text { Outcomes ( } p \text { values); } \\
\text { conclusions }\end{array}$ & $\begin{array}{l}\text { Ranking } \\
\text { of study } \\
\text { design }^{1}\end{array}$ & $\mathrm{COI}$ \\
\hline $\begin{array}{l}\text { Egglestone } \\
{[8], 2013}\end{array}$ & $\begin{array}{l}\text { Adults who had } \\
\text { purchased a } \\
\text { DTC genetic } \\
\text { test or were } \\
\text { considering } \\
\text { purchasing a } \\
\text { test or who were } \\
\text { awaiting their } \\
\text { results } \\
(n=275)\end{array}$ & $\begin{array}{l}\text { Genetic } \\
\text { testing }\end{array}$ & $\begin{array}{l}\text { No genetic } \\
\text { testing }\end{array}$ & NR (NR) & Varied & $\begin{array}{l}\text { Nutrition (health- } \\
\text { ier diet, vitamins/ } \\
\text { supplements, } \\
\text { caffeine, fibre, } \\
\text { salt, fat, and } \\
\text { fruits/vegetables), } \\
\text { physical activity, } \\
\text { and smoking }\end{array}$ & $\begin{array}{l}\text { Greater health behaviour } \\
\text { scores in the genetic } \\
\text { testing group vs. the } \\
\text { control group ( } p=0.02 \\
\text { for pooled nutrition, } \\
\text { physical activity, and } \\
\text { smoking); the most } \\
\text { common changes were } \\
\text { "healthier diet," "more } \\
\text { exercise," and "taking } \\
\text { vitamins or supple- } \\
\text { ments"; more often } \\
\text { reported "sufficient fruit } \\
\text { and vegetable intake" in } \\
\text { the genetic testing group } \\
\text { ( } p=0.03 \text { ); NS changes in } \\
\text { smoking individually }\end{array}$ & 2 & NR \\
\hline $\begin{array}{l}\text { Bloss [48], } \\
2013\end{array}$ & $\begin{array}{l}\text { Adults working } \\
\text { at health and } \\
\text { technology } \\
\text { companies } \\
(n=3,639 ; \\
n=2,037 \text { at } \\
3 \text { months, } n= \\
1,325 \text { at } 14 \pm \\
1.3 \text { months })\end{array}$ & $\begin{array}{l}\text { Genetic } \\
\text { testing }\end{array}$ & None & $\begin{array}{l}\text { Deep vein thrombo- } \\
\text { sis, melanoma, } \\
\text { sarcoidosis, haemo- } \\
\text { chromatosis, lactose } \\
\text { intolerance, breast } \\
\text { cancer, prostate } \\
\text { cancer }+20 \text { other } \\
\text { conditions not listed } \\
\text { (variable) }\end{array}$ & $\begin{array}{l}3 \text { months } \\
14 \pm 1.3 \\
\text { months }\end{array}$ & $\begin{array}{l}\text { Nutrition (dietary } \\
\text { fat) and physical } \\
\text { activity }\end{array}$ & $\begin{array}{l}\text { NS changes in nutrition } \\
\text { or physical activity at } \\
3 \text { months (significance } \\
\text { level NR) or } 14 \pm 1.3 \\
\text { months }\end{array}$ & 4 & No \\
\hline $\begin{array}{l}\text { Kaufman [49], } \\
2012\end{array}$ & $\begin{array}{l}\text { Adult } \\
\text { customers of } \\
\text { DTC genetic } \\
\text { testing } \\
\text { companies } \\
(n=3,167 ; \\
n=1,048)\end{array}$ & $\begin{array}{l}\text { Genetic } \\
\text { testing } \\
\text { and } \\
\text { High-risk } \\
\text { genetic } \\
\text { result }\end{array}$ & $\begin{array}{l}\text { Non-risk } \\
\text { genetic result }\end{array}$ & $\begin{array}{l}\text { Variable } \\
\text { (variable) }\end{array}$ & 2-8 months & $\begin{array}{l}\text { Nutrition (change } \\
\text { diet) and physical } \\
\text { activity }\end{array}$ & $\begin{array}{l}\text { The participants who } \\
\text { considered themselves at } \\
\text { high risk of colon cancer } \\
\text { were significantly more } \\
\text { likely to change their diet } \\
\text { ( } p=0.02) \text { and start } \\
\text { exercising more ( } p= \\
0.01 \text { ) than those who } \\
\text { considered themselves at } \\
\text { low risk of colon cancer; } \\
10 \% \text { of all participants } \\
\text { reported they changed a } \\
\text { supplement, } 33 \% \text { report- } \\
\text { ed being more careful } \\
\text { about their diet, and } 14 \% \\
\text { reported exercising more }\end{array}$ & 2 & NR \\
\hline $\begin{array}{l}\text { Hollands [50], } \\
2012\end{array}$ & $\begin{array}{l}\text { Adults with } \\
\text { 1st-degree } \\
\text { relatives with } \\
\text { Crohn disease } \\
(n=497 \\
n=426)\end{array}$ & $\begin{array}{l}\text { Genetic } \\
\text { testing } \\
\text { and } \\
\text { High-risk } \\
\text { genetic } \\
\text { result }\end{array}$ & $\begin{array}{l}\text { No genetic } \\
\text { testing } \\
\text { and } \\
\text { Non-risk } \\
\text { genetic result }\end{array}$ & $\begin{array}{l}\text { Crohn disease } \\
\text { (NOD2) }\end{array}$ & 6 months & Smoking & $\begin{array}{l}\text { NS changes in smoking } \\
\text { cessation between the } \\
\text { genetic testing and the } \\
\text { no-genetic-testing group; } \\
\text { NS changes in smoking } \\
\text { cessation between the } \\
\text { high-risk and the non- } \\
\text { risk genetic result group } \\
\text { (significance level NR) }\end{array}$ & 1 & No \\
\hline $\begin{array}{l}\text { Bloss [36], } \\
2011\end{array}$ & $\begin{array}{l}\text { Adults working } \\
\text { at health and } \\
\text { technology } \\
\text { companies } \\
(n=3,639 ; \\
n=2,037)\end{array}$ & $\begin{array}{l}\text { Genetic } \\
\text { testing }\end{array}$ & None & $\begin{array}{l}23 \text { conditions } \\
\text { including breast } \\
\text { and prostate } \\
\text { cancer (NR) }\end{array}$ & $\begin{array}{l}5.6 \pm 2.4 \\
\text { months }\end{array}$ & $\begin{array}{l}\text { Nutrition (dietary } \\
\text { fat) and physical } \\
\text { activity }\end{array}$ & $\begin{array}{l}\text { NS changes in nutrition } \\
\text { and/or physical activity } \\
\text { following genetic testing }\end{array}$ & 4 & NR \\
\hline $\begin{array}{l}\text { Vernarelli } \\
\text { [51], } 2010\end{array}$ & $\begin{array}{l}\text { Adults with at } \\
\text { least one parent } \\
\text { who developed } \\
\text { Alzheimer } \\
\text { disease } \\
(n=279 \\
n=272)\end{array}$ & $\begin{array}{l}\text { High-risk } \\
\text { genetic } \\
\text { result }\end{array}$ & $\begin{array}{l}\text { Non-risk } \\
\text { genetic result }\end{array}$ & $\begin{array}{l}\text { Alzheimer } \\
\text { disease (apoE) }\end{array}$ & 6 weeks & $\begin{array}{l}\text { Nutrition (dietary } \\
\text { supplement use) } \\
\text { and physical } \\
\text { activity }\end{array}$ & $\begin{array}{l}\text { The high-risk genetic } \\
\text { result group was more } \\
\text { likely to take supple- } \\
\text { ments than the non-risk } \\
\text { genetic result group } \\
(p=0.0001)\end{array}$ & 3 & No \\
\hline
\end{tabular}


Table 1 (continued)

\begin{tabular}{|c|c|c|c|c|c|c|c|c|c|}
\hline $\begin{array}{l}\text { First author } \\
\text { [Ref.], date }\end{array}$ & $\begin{array}{l}\text { Participants } \\
\text { ( } n \text { baseline; } \\
n \text { follow-up) }\end{array}$ & $\begin{array}{l}\text { Interven- } \\
\text { tion } \\
\text { group(s) }\end{array}$ & $\begin{array}{l}\text { Comparison } \\
\operatorname{group}(\mathrm{s})\end{array}$ & $\begin{array}{l}\text { Target diseases/ } \\
\text { conditions } \\
\text { (genes tested) }\end{array}$ & Follow-up & $\begin{array}{l}\text { Lifestyle habits } \\
\text { assessed }\end{array}$ & $\begin{array}{l}\text { Outcomes ( } p \text { values); } \\
\text { conclusions }\end{array}$ & $\begin{array}{l}\text { Ranking } \\
\text { of study } \\
\text { design }^{1}\end{array}$ & $\mathrm{COI}$ \\
\hline $\begin{array}{l}\text { Hishida [52], } \\
2010\end{array}$ & $\begin{array}{l}\text { Adult smokers } \\
(n=562 ; \\
n=533)\end{array}$ & $\begin{array}{l}\text { Genetic } \\
\text { testing }\end{array}$ & $\begin{array}{l}\text { No genetic } \\
\text { testing }\end{array}$ & $\begin{array}{l}\text { Lung and } \\
\text { oesophageal cancer } \\
(\mathrm{L}-m y c)\end{array}$ & 12 months & Smoking & $\begin{array}{l}\text { NS changes in smoking } \\
\text { cessation between the } \\
\text { genetic testing and the } \\
\text { no-genetic-testing group }\end{array}$ & 1 & No \\
\hline $\begin{array}{l}\text { Quach [53], } \\
2009\end{array}$ & $\begin{array}{l}\text { Adults with a } \\
\text { personal and/or } \\
\text { family history of } \\
\text { breast and/or } \\
\text { ovarian cancer } \\
(n=120 ; \mathrm{NR})\end{array}$ & $\begin{array}{l}\text { Genetic } \\
\text { testing }\end{array}$ & None & $\begin{array}{l}\text { Breast and ovarian } \\
\text { cancer (BRCA1/2) }\end{array}$ & 6 months & $\begin{array}{l}\text { Nutrition (healthy } \\
\text { diet and vitamin } \\
\text { use) and physical } \\
\text { activity }\end{array}$ & $\begin{array}{l}\text { NS changes in nutrition, } \\
\text { vitamin use, or physical } \\
\text { activity after genetic } \\
\text { testing (significance level } \\
\text { NR) }\end{array}$ & 4 & NR \\
\hline $\begin{array}{l}\text { O’Neill [54], } \\
2008\end{array}$ & $\begin{array}{l}\text { Adult females } \\
\text { (NR; } n=115 \text { at } \\
1 \text { month and } \\
6 \text { months) }\end{array}$ & $\begin{array}{l}\text { High-risk } \\
\text { genetic } \\
\text { result }\end{array}$ & $\begin{array}{l}\text { Uninforma- } \\
\text { tive genetic } \\
\text { result } \\
\text { and } \\
\text { Non-risk } \\
\text { genetic result }\end{array}$ & $\begin{array}{l}\text { Breast cancer } \\
(\text { BRCA } 1 / 2)\end{array}$ & $\begin{array}{l}1 \text { month } \\
6 \text { months }\end{array}$ & $\begin{array}{l}\text { Nutrition (satu- } \\
\text { rated fat, fruit/ } \\
\text { vegetables) and } \\
\text { physical activity }\end{array}$ & $\begin{array}{l}\text { NS differences between } \\
\text { groups in nutrition or } \\
\text { physical activity at } \\
\text { baseline and } 1 \text { month or } \\
6 \text { months following } \\
\text { genetic testing }\end{array}$ & 3 & NR \\
\hline $\begin{array}{l}\text { Chao [55], } \\
2008\end{array}$ & $\begin{array}{l}\text { Adult with } \\
\text { parent who } \\
\text { developed } \\
\text { Alzheimer } \\
\text { disease } \\
(n=162 ; \\
n=147)\end{array}$ & $\begin{array}{l}\text { Genetic } \\
\text { testing } \\
\text { and } \\
\text { High-risk } \\
\text { genetic } \\
\text { result }\end{array}$ & $\begin{array}{l}\text { No genetic } \\
\text { testing } \\
\text { and } \\
\begin{array}{l}\text { Non-risk } \\
\text { genetic result }\end{array}\end{array}$ & $\begin{array}{l}\text { Alzheimer disease } \\
\text { (apoE) }\end{array}$ & 12 months & $\begin{array}{l}\text { Nutrition (chang- } \\
\text { es in diet, changes } \\
\text { in vitamin/ } \\
\text { supplement use) } \\
\text { and physical } \\
\text { activity }\end{array}$ & $\begin{array}{l}\text { The high-risk genetic } \\
\text { result group was more } \\
\text { likely to report a nutri- } \\
\text { tion or physical activity } \\
\text { change than the non-risk } \\
\text { genetic result group ( } p= \\
0.003 \text { ) and the no-genet- } \\
\text { ic-testing group ( } p= \\
0.03) \text {; most common was } \\
\text { a change in medication/ } \\
\text { supplement use (specifi- } \\
\text { cally vitamin E) }\end{array}$ & 1 & NR \\
\hline $\begin{array}{l}\text { Sanderson } \\
{[56], 2008}\end{array}$ & $\begin{array}{l}\text { Adult smokers } \\
(\mathrm{NR} ; n=61)\end{array}$ & $\begin{array}{l}\text { Genetic } \\
\text { testing } \\
\text { and } \\
\text { High-risk } \\
\text { genetic } \\
\text { result }\end{array}$ & $\begin{array}{l}\text { No genetic } \\
\text { testing } \\
\text { and } \\
\text { Non-risk } \\
\text { genetic result }\end{array}$ & $\begin{array}{l}\text { Lung cancer } \\
\text { (GSTM1) }\end{array}$ & $\begin{array}{l}1 \text { week } \\
2 \text { months }\end{array}$ & Smoking & $\begin{array}{l}\text { Fewer cigarettes smoked } \\
\text { ( } p=0.009) \text { and greater } \\
\text { quit rates }(p=0.009) \text { at } \\
\text { the 1-week follow-up in } \\
\text { the high-risk genetic } \\
\text { result group than in the } \\
\text { no-genetic-testing group; } \\
\text { NS differences at the } \\
\text { 2-month follow-up } \\
\text { between the groups for } \\
\text { cigarettes smoked and } \\
\text { quit rates }\end{array}$ & 1 & No \\
\hline $\begin{array}{l}\text { Rees [57], } \\
2007\end{array}$ & $\begin{array}{l}\text { Adult females } \\
(n=23)\end{array}$ & $\begin{array}{l}\text { Genetic } \\
\text { testing }\end{array}$ & None & $\begin{array}{l}\text { Breast cancer } \\
(\text { BRCA } 1 / 2)\end{array}$ & $\begin{array}{l}\text { Varied - up } \\
\text { to } 18 \text { months }\end{array}$ & $\begin{array}{l}\text { Nutrition (dietary } \\
\text { changes), physical } \\
\text { activity, and } \\
\text { smoking }\end{array}$ & $\begin{array}{l}\text { Few women reported a } \\
\text { significant impact on } \\
\text { nutrition, physical } \\
\text { activity, and/or smoking } \\
\text { as a result of receiving } \\
\text { genetic testing results } \\
\text { and counselling (signifi- } \\
\text { cance level not applica- } \\
\text { ble) }\end{array}$ & $\begin{array}{l}\text { Qualita- } \\
\text { tive }\end{array}$ & NR \\
\hline Rief [58], 2007 & $\begin{array}{l}\text { Adults } \\
(n=294)\end{array}$ & $\begin{array}{l}\text { Genetic } \\
\text { testing } \\
\text { and } \\
\text { consulta- } \\
\text { tion }\end{array}$ & $\begin{array}{l}\begin{array}{l}\text { No genetic } \\
\text { testing - } \\
\text { consultation } \\
\text { only }\end{array} \\
\text { and } \\
\text { No genetic } \\
\text { testing } \\
\text { and no } \\
\text { consultation }\end{array}$ & Obesity (NR) & 6 months & $\begin{array}{l}\text { Nutrition } \\
\text { (restraint eating) }\end{array}$ & $\begin{array}{l}\text { NS changes to restraint } \\
\text { eating in the genetic } \\
\text { testing group compared } \\
\text { to the no-genetic-testing } \\
\text { groups }\end{array}$ & 1 & No \\
\hline
\end{tabular}


Table 1 (continued)

\begin{tabular}{|c|c|c|c|c|c|c|c|c|c|}
\hline $\begin{array}{l}\text { First author } \\
\text { [Ref.], date }\end{array}$ & $\begin{array}{l}\text { Participants } \\
\text { ( } n \text { baseline; } \\
n \text { follow-up) }\end{array}$ & $\begin{array}{l}\text { Interven- } \\
\text { tion } \\
\text { group(s) }\end{array}$ & $\begin{array}{l}\text { Comparison } \\
\text { group }(s)\end{array}$ & $\begin{array}{l}\text { Target diseases/ } \\
\text { conditions } \\
\text { (genes tested) }\end{array}$ & Follow-up & $\begin{array}{l}\text { Lifestyle habits } \\
\text { assessed }\end{array}$ & $\begin{array}{l}\text { Outcomes ( } p \text { values); } \\
\text { conclusions }\end{array}$ & $\begin{array}{l}\text { Ranking } \\
\text { of study } \\
\text { design }^{1}\end{array}$ & $\mathrm{COI}$ \\
\hline $\begin{array}{l}\text { Carpenter } \\
{[59], 2007}\end{array}$ & $\begin{array}{l}\text { Adult smokers } \\
(\mathrm{n}=729 \\
n=199)\end{array}$ & $\begin{array}{l}\text { High-risk } \\
\text { genetic } \\
\text { result }\end{array}$ & $\begin{array}{l}\text { Non-risk } \\
\text { genetic result }\end{array}$ & Emphysema (AAT) & 3 months & Smoking & $\begin{array}{l}\text { Those with high-risk } \\
\text { genetic results made } \\
\text { significantly greater quit } \\
\text { attempts than the non- } \\
\text { risk genetic result group } \\
(p=0.004)\end{array}$ & 3 & NR \\
\hline Ito [60], 2006 & $\begin{array}{l}\text { Adult smokers } \\
\text { ( } n=697 ; n= \\
369 \text { with data } \\
\text { for baseline, } \\
3 \text { and } 9 \text { months) }\end{array}$ & $\begin{array}{l}\text { Genetic } \\
\text { testing }\end{array}$ & $\begin{array}{l}\text { No genetic } \\
\text { testing }\end{array}$ & $\begin{array}{l}\text { Lung and } \\
\text { oesophageal cancer } \\
(\mathrm{L}-m y c)\end{array}$ & $\begin{array}{l}3 \text { months } \\
9 \text { months }\end{array}$ & Smoking & $\begin{array}{l}\text { NS differences in smok- } \\
\text { ing cessation between } \\
\text { groups at } 3 \text { months } \\
\text { (significance level NR) } \\
\text { or } 9 \text { months }\end{array}$ & 1 & NR \\
\hline $\begin{array}{l}\text { Marteau [61], } \\
2004\end{array}$ & $\begin{array}{l}\text { Adult probands } \\
\text { and their adult } \\
\text { relatives with } \\
\text { familial hyper- } \\
\text { cholesterolae- } \\
\text { mia }(n=341 ; \\
n=275)\end{array}$ & $\begin{array}{l}\text { Genetic } \\
\text { testing }\end{array}$ & $\begin{array}{l}\text { No genetic } \\
\text { testing }\end{array}$ & $\begin{array}{l}\text { Familial } \\
\text { hypercholes- } \\
\text { terolaemia (NR) }\end{array}$ & 6 months & $\begin{array}{l}\text { Nutrition (total } \\
\text { fat and unsaturat- } \\
\text { ed fat), physical } \\
\text { activity, and } \\
\text { smoking }\end{array}$ & $\begin{array}{l}\text { NS impact on nutrition, } \\
\text { physical activity, or } \\
\text { smoking with genetic } \\
\text { testing }\end{array}$ & 1 & NR \\
\hline $\begin{array}{l}\text { McBride [62], } \\
2002\end{array}$ & $\begin{array}{l}\text { Adult smokers } \\
(n=557 ; \\
n=412 \text { at } \\
6 \text { months, } \\
n=356 \text { at } \\
12 \text { months, } \\
n=487 \text { with } \\
\text { data from all } 3 \\
\text { time points) }\end{array}$ & $\begin{array}{l}\text { Genetic } \\
\text { testing }\end{array}$ & $\begin{array}{l}\text { No genetic } \\
\text { testing }\end{array}$ & $\begin{array}{l}\text { Lung cancer } \\
\text { (GSTM1) }\end{array}$ & $\begin{array}{l}6 \text { months } \\
12 \text { months }\end{array}$ & Smoking & $\begin{array}{l}\text { Greater smoking cessa- } \\
\text { tion in the genetic testing } \\
\text { group than in the no- } \\
\text { genetic-testing group } \\
(p<0.006) \text { at } 6 \text { months; } \\
\text { NS smoking cessation } \\
\text { rates at } 12 \text { months }\end{array}$ & 1 & NR \\
\hline $\begin{array}{l}\text { Audrain [24], } \\
1997\end{array}$ & $\begin{array}{l}\text { Adult smokers } \\
(n=550 \\
n=426)\end{array}$ & $\begin{array}{l}\text { Genetic } \\
\text { testing }\end{array}$ & $\begin{array}{l}\text { No genetic } \\
\text { testing }\end{array}$ & $\begin{array}{l}\text { Lung cancer } \\
\text { (CYP2D6) }\end{array}$ & 12 months & Smoking & $\begin{array}{l}\text { Greater likelihood of quit } \\
\text { attempts in the genetic } \\
\text { testing group than in the } \\
\text { no-genetic-testing group } \\
(p=0.02) \text {; NS change in } \\
\text { 30-day cessation between } \\
\text { groups }\end{array}$ & 1 & NR \\
\hline
\end{tabular}

COI, conflict of interest; EPA, eicosapentaenoic acid; DHA, docosahexaenoic acid; NS, not statistically significant ( $p>0.05$ unless otherwise stated); NR, not reported; DTC, direct to consumer; MUFA, monounsaturated fatty acid; PUFA, polyunsaturated fatty acid. ${ }^{1}$ The rank of the study design is as follows, based on the categories of the NIH Quality Assessment Tools [29] in combination with consideration of the hierarchy of evidence [63]: 1 = controlled intervention study; $2=$ observational cohort/cross-sectional study; $3=$ case-control study; 4 = pre-post study with no control group. ${ }^{2}$ Note: significance levels for this group of participants are reported in Bloss et al. [36].

cerning, since overall the ratings were "poor" and only 6 of the 26 studies (23\%) received a "good" rating. Thus, it is clear that the studies did not provide high-quality interventions to their participants, which helps to explain why the majority of studies did not report that genetic interventions facilitated lifestyle behaviour change.

\section{Are We Considering Validated Behaviour \\ Change Theory?}

Consideration of the TPB and/or one or more of the theory's three key components had mode overall ratings of "poor." The deductive content analyses of the theoretical underpinnings mentioned in the studies are summarized in online supplementary Table 2 . Fifteen studies (58\%) did not make reference to any specific behaviour change theory or model within the text. When a theory was included, it was generally only briefly mentioned and was not thoroughly incorporated into the study design, or expanded upon in the discussion. No study specifically referred to the TPB, suggesting that researchers have yet to consider this important theory in their study design or interpretation of findings. Several studies incidentally considered certain aspects of the TPB in the development of their scientific methods or within the text, such as the consideration of behavioural control by assessing one or more social determinants of health, such as income [64]. Overall, behaviour change theory is not being thoroughly incorporated into genetic testing behaviour change research. 
Table 2. Summary of quality assessment ratings and impact of genetic testing on lifestyle factor(s) of interest

\begin{tabular}{|c|c|c|c|c|c|c|c|c|c|c|c|}
\hline \multirow{2}{*}{$\begin{array}{l}\text { Ranking } \\
\text { of study } \\
\text { design }\end{array}$} & \multirow[t]{2}{*}{$\begin{array}{l}\text { First author } \\
\text { [Ref.], year }\end{array}$} & \multicolumn{4}{|c|}{ Quality assessment rating } & \multicolumn{4}{|c|}{$\begin{array}{l}\text { Key findings: impact of genetic testing on lifestyle } \\
\text { factor(s) of interest }\end{array}$} & \multirow{2}{*}{$\begin{array}{l}\text { Source of } \\
\text { genetic } \\
\text { information }\end{array}$} & \multirow{2}{*}{$\begin{array}{l}\text { Specific lifestyle } \\
\text { factors with significant } \\
\text { improvement }\end{array}$} \\
\hline & & methods & $\begin{array}{l}\text { genetic } \\
\text { info }\end{array}$ & ТPB & $\begin{array}{l}\text { overall } \\
\text { quality } \\
\text { score }\end{array}$ & nutrition & $\mathrm{PA}$ & smoking & $\begin{array}{ll}\text { nutrition } & \text { nutrition, } \\
\text { and PA } & \text { PA, and } \\
& \text { smoking }\end{array}$ & & \\
\hline 1 & Roke [43], 2017 & Good & Fair & Fair & 7 & $A$ & & & & Other & N/A \\
\hline 1 & $\begin{array}{l}\text { Hietaranta-Luoma } \\
{[9], 2015}\end{array}$ & Fair & Good & Poor & 6 & $\begin{array}{l}\checkmark^{\mathrm{a}} \\
\text { (2 weeks) } \\
\checkmark^{\mathrm{b}} \\
\text { ( } 6 \text { months) } \\
\sqrt{\mathrm{c}}^{\mathrm{c}} \\
\text { (12 months) }\end{array}$ & $A$ & & & $\mathrm{HCP}$ & $\begin{array}{l}\text { Improved dietary fat } \\
\text { quality (high-risk genotype } \\
\text { vs. control at } 2 \text { weeks and } \\
\text { baseline to 6-month } \\
\text { follow-up in high-risk } \\
\text { genotype group); } \\
\text { decreased intake of high- } \\
\text { fat, high-sugar foods (in } \\
\text { low-risk genotype vs. } \\
\text { control at } 12 \text { months) }\end{array}$ \\
\hline 1 & Marsaux [47], 2015 & Fair & Fair & Poor & 5 & & $A$ & & & DTC & N/A \\
\hline 1 & Meisel [44], 2015 & Poor & Fair & Fair & 5 & $A$ & $A$ & & & DTC & N/A \\
\hline 1 & Voils [46], 2015 & Fair & Good & Poor & 6 & $\begin{array}{l}\checkmark \mathrm{d} \\
(3 \text { months }) \\
\Delta \\
\text { (6 months })\end{array}$ & $A$ & & & $\mathrm{HCP}$ & $\begin{array}{l}\text { Reduced calories and fat } \\
\text { (MUFA and PUFA) }\end{array}$ \\
\hline 1 & Nielsen [11], 2014 & Good & Fair & Poor & 6 & $\begin{array}{l}\Delta \\
\text { (3 months) } \\
\sqrt{ } \text { a } \\
\text { (12 months })\end{array}$ & & & & DTC & Reduced sodium intake \\
\hline 1 & Hollands [50], 2012 & Good & Good & Poor & 7 & & & $\Delta$ & & Other & N/A \\
\hline 1 & Hishida [52], 2010 & Poor & Poor & Poor & 3 & & & $\Delta$ & & $\mathrm{HCP}$ & N/A \\
\hline 1 & Chao [55], 2008 & Fair & Poor & Poor & 4 & $A$ & $A$ & & $\checkmark \mathrm{a}, \mathrm{e}$ & $\mathrm{HCP}$ & $\begin{array}{l}\text { General improvements to } \\
\text { nutrition and PA; vitamin } \\
\text { E supplementation was the } \\
\text { most common change } \\
\text { reported }\end{array}$ \\
\hline 1 & Sanderson [56], 2008 & Poor & Fair & Fair & 5 & & & $\begin{array}{l}\checkmark^{\mathrm{a}} \\
(1 \text { week }) \\
\triangle \\
\text { (2 months) }\end{array}$ & & $\mathrm{HCP}$ & $\begin{array}{l}\text { Fewer cigarettes smoked } \\
\text { and greater smoking } \\
\text { cessation }\end{array}$ \\
\hline 1 & Rief [58], 2007 & Fair & Good & Poor & 6 & $\Delta$ & & & & $\mathrm{HCP}$ & N/A \\
\hline 1 & Ito [60], 2006 & Poor & Good & Fair & 6 & & & $A$ & & Other & N/A \\
\hline 1 & Marteau [61], 2004 & Fair & Fair & Fair & 6 & $\Delta$ & $\Delta$ & $\Delta$ & & $\mathrm{HCP}$ & N/A \\
\hline 1 & McBride [62], 2002 & Poor & Good & Fair & 6 & & & $\begin{array}{l}\checkmark \mathrm{d} \\
(6 \text { months }) \\
\triangle \\
(12 \\
\text { months })\end{array}$ & & Other & Greater smoking cessation \\
\hline 1 & Audrain [24], 1997 & Fair & Fair & Fair & 6 & & & $\begin{array}{l}\checkmark \text { d (quit } \\
\text { attempts) } \\
\triangle(30 \text {-day } \\
\text { cessation) }\end{array}$ & & $\mathrm{HCP}$ & $\begin{array}{l}\text { Greater likelihood of quit } \\
\text { attempts }\end{array}$ \\
\hline \multicolumn{2}{|c|}{$\operatorname{Summary}^{2}(n=15)$} & Fair & Fair & Poor & 5.6 & $3 / 8$ & $0 / 6$ & $3 / 7$ & $1 / 1$ & & \\
\hline 2 & Egglestone [8], 2013 & Poor & Poor & Poor & 3 & $\checkmark^{d}$ & $\checkmark^{\mathrm{d}}$ & $\Delta$ & $\checkmark \mathrm{d}$ & DTC & $\begin{array}{l}\text { Greater health behaviour } \\
\text { scores; the most common } \\
\text { changes were "healthier } \\
\text { diet," "more exercise," and } \\
\text { "taking vitamins or } \\
\text { supplements"; more often } \\
\text { reported "sufficient fruit } \\
\text { and vegetable intake" }\end{array}$ \\
\hline
\end{tabular}


Table 2 (continued)

\begin{tabular}{|c|c|c|c|c|c|c|c|c|c|c|c|}
\hline \multirow{2}{*}{$\begin{array}{l}\text { Ranking } \\
\text { of study } \\
\text { design }^{1}\end{array}$} & \multirow[t]{2}{*}{$\begin{array}{l}\text { First author } \\
\text { [Ref.], year }\end{array}$} & \multicolumn{4}{|c|}{ Quality assessment rating } & \multicolumn{4}{|c|}{$\begin{array}{l}\text { Key findings: impact of genetic testing on lifestyle } \\
\text { factor(s) of interest }\end{array}$} & \multirow{2}{*}{$\begin{array}{l}\text { Source of } \\
\text { genetic } \\
\text { information }\end{array}$} & \multirow{2}{*}{$\begin{array}{l}\text { Specific lifestyle } \\
\text { factors with significant } \\
\text { improvement }\end{array}$} \\
\hline & & methods & $\begin{array}{l}\text { genetic } \\
\text { info }\end{array}$ & TPB & $\begin{array}{l}\text { overall } \\
\text { quality } \\
\text { score }\end{array}$ & nutrition & PA smoking & $\begin{array}{l}\text { nutrition } \\
\text { and PA }\end{array}$ & $\begin{array}{l}\text { nutrition, } \\
\text { PA, and } \\
\text { smoking }\end{array}$ & & \\
\hline 2 & Kaufman [49], 2012 & Fair & Poor & Poor & 4 & $\checkmark \mathrm{e}$ & $\sqrt{ } \mathrm{e}$ & & & $\begin{array}{l}\text { DTC }+ \\
\text { optional HCP }\end{array}$ & $\begin{array}{l}\text { "Changed their diet" and } \\
\text { "started exercising more" }\end{array}$ \\
\hline \multicolumn{2}{|c|}{$\operatorname{Summary}^{2}(n=2)$} & $\begin{array}{l}\text { Fair - } \\
\text { poor }\end{array}$ & Poor & Poor & 3.5 & $2 / 2$ & $2 / 2 \quad 0 / 1$ & & $1 / 1$ & & \\
\hline 3 & Marsaux [10], 2016 & Fair & Fair & Poor & 5 & & $\Delta$ & & & DTC & N/A \\
\hline 3 & Vernarelli [51], 2010 & Good & Poor & Poor & 5 & $\sqrt{ } \mathrm{e}$ & $\Delta$ & & & HCP & $\begin{array}{l}\text { Greater changes in } \\
\text { supplement use; vitamin } \mathrm{E} \text {, } \\
\text { vitamin } \mathrm{C} \text {, botanicals, } \\
\text { multivitamins, vitamin } \mathrm{B}, \\
\text { and fish oil/omega were } \\
\text { the most common changes } \\
\text { reported }\end{array}$ \\
\hline 3 & O’Neill [54], 2008 & Good & Poor & Poor & 5 & $\Delta$ & $\Delta$ & & & $\mathrm{HCP}$ & N/A \\
\hline 3 & Carpenter [59], 2007 & Fair & Fair & Poor & 5 & & $\checkmark$ e & & & $\begin{array}{l}\text { DTC }+ \\
\text { optional HCP }\end{array}$ & Greater 24-h quit attempts \\
\hline \multicolumn{2}{|c|}{$\operatorname{Summary}^{2}(n=4)$} & $\begin{array}{l}\text { Good - } \\
\text { fair }\end{array}$ & $\begin{array}{l}\text { Fair - } \\
\text { poor }\end{array}$ & Poor & 5.0 & $1 / 2$ & $0 / 3 \quad 1 / 1$ & & & & \\
\hline 4 & Boeldt [45], 2015 & Fair & Poor & Fair & 5 & $\Delta$ & $\Delta$ & & & $\begin{array}{l}\text { DTC + } \\
\text { optional HCP }\end{array}$ & N/A \\
\hline 4 & Bloss [48], 2013 & Fair & Poor & Fair & 5 & $\Delta$ & $A$ & & & $\begin{array}{l}\text { DTC }+ \\
\text { optional HCP }\end{array}$ & N/A \\
\hline 4 & Bloss [36], 2011 & Fair & Poor & Poor & 4 & $A$ & $A$ & & & $\begin{array}{l}\mathrm{DTC}+ \\
\text { optional HCP }\end{array}$ & N/A \\
\hline 4 & Quach [53], 2009 & Fair & Poor & Fair & 5 & $\Delta$ & $\Delta$ & & & $\mathrm{HCP}$ & N/A \\
\hline \multicolumn{2}{|c|}{ Summary $^{2}(n=4)$} & Fair & Poor & Fair & 4.8 & $0 / 4$ & $0 / 4$ & & & & \\
\hline Qualitative & Rees [57], 2007 & Good & Poor & Poor & 5 & $\Delta$ & $\Delta$ & & & $\mathrm{HCP}$ & N/A \\
\hline \multicolumn{2}{|c|}{$\operatorname{Summary}^{2}(n=1)$} & Good & Poor & Poor & 5.0 & $0 / 1$ & $0 / 1$ & & & & \\
\hline \multicolumn{2}{|c|}{$\begin{array}{l}\text { Summary of all studies } \\
(n=26)\end{array}$} & FAIR & POOR & POOR & 5.2 & \multicolumn{4}{|c|}{$\begin{array}{l}\text { Nutrition: 6/18 (33\%) } \\
\text { PA: } 2 / 16(13 \%) \\
\text { Smoking: 4/12 (33\%) }\end{array}$} & \multicolumn{2}{|c|}{$\begin{array}{l}\text { Studies with significant beneficial } \\
\text { health behaviour change(s): } \\
7 / 93(78 \%) \text { provided actionable } \\
\text { recommendations } \\
\text { Studies with null findings: } \\
\text { 7/14 }(50 \%) \text { provided actionable } \\
\text { recommendations }\end{array}$} \\
\hline
\end{tabular}

"Other" sources of genetic information: Roke et al. [43], 2017, used a researcher; McBride et al. [62], 2002, used "trained counsellors"; Ito et al. [60], 2006, used a "trained interviewer"; Hollands et al. [50], 2009, used a "trained research counsellor.” No studies found a detrimental effect of genetic testing on lifestyle change. $\checkmark$, statistically significant beneficial behaviour change(s); $A$, no statistically significant behaviour change(s); blank cells, lifestyle factor(s) of interest was/were not assessed; N/A, not applicable; TPB, theory of planned behaviour; PA, physical activity; HCP, genetic intervention offered through a healthcare provider; DTC, genetic intervention offered direct to consumer; Other, another method was used to deliver the genetic intervention to the participants; MUFA, monounsaturated fatty acid; PUFA, polyunsaturated fatty acid. ${ }^{1}$ The rank of the study design is as follows, based on the National Institutes of Health Quality Assessment Tools [29] and the hierarchy of evidence pyramid [63]: $1=$ controlled intervention study; $2=$ observational cohort/cross-sectional study; 3 = case-control study; $4=$ pre-post study with no control group. ${ }^{2} n=\mathrm{x}$ indicates the total number of studies included in the summary; modes are reported for each of the three quality assessment ratings; $x / x$ indicates the number of beneficial behaviour change findings/the total number of studies (note: several studies included multiple analyses such as those with more than one follow-up time point, and those assessing more than one lifestyle factor of interest); the overall quality score is represented as a mean. ${ }^{3}$ Three studies did not provide information about whether or not actionable recommendations were provided. ${ }^{a}$ High-risk genotype vs. control group. ${ }^{\mathrm{b}}$ Baseline to 6 -month follow-up in high-risk genotype group. ${ }^{\mathrm{c}}$ Non-risk genotype vs. control group. ${ }^{\mathrm{d}}$ Genetic testing group vs. control group. ${ }^{\mathrm{e}}$ High-risk genotype group vs. non-risk genotype group. 
Does Genetic Testing Impact Changes in Nutrition, Physical Activity, and/or Smoking Behaviour?

Overall. Given the heterogeneity of the literature and complexity of genetics-based behaviour change research, a cause-and-effect relationship between genetic testing and health behaviour change cannot be identified. Notably, it appears that it is unlikely that genetic testing has a "fatalistic" or negative impact on health behaviour change related to nutrition, physical activity, and smoking, since no study found that genetic testing negatively impacted the health behaviours of interest to the present review. Interestingly, $78 \%$ of the studies with health-promoting lifestyle behaviour change findings provided their participants with a genetics-based intervention that included actionable health behaviour recommendations. Examples of actionable recommendations provided to participants for each lifestyle factor included recommendations to reduce sodium intake (nutrition) [11], incorporate exercise into one's daily routine (physical activity) [44], and quit smoking (smoking) [24]. Conversely, only $50 \%$ of the studies with null findings provided their participants with actionable health behaviour recommendations. Since an overarching cause-and-effect statement about the impact of genetic testing on behaviour change cannot be made, a best evidence synthesis is provided below.

Nutrition. Of the 18 articles that assessed a nutritionrelated outcome, 6 (33\%) showed a positive, health-promoting effect of genetic testing on behaviour change at one or more time points (both short term and long term, as further outlined in Tables 1 and 2). While this does not indicate that the majority of studies positively influenced nutrition, multiple studies of good quality have demonstrated that it is possible to facilitate healthier nutritional behaviours through the provision of genetic testing $[8,9$, $11,46,49,51]$.

Physical Activity. The provision of genetic testing to facilitate physical activity behaviour change does not appear to be as promising as behaviour change related to nutrition. Of the 16 studies that analysed physical activity-related outcomes independently, only 2 (13\%) found positive influences of genetic testing on physical activity $[8,49]$, with follow-up periods ranging from 2 to 8 months in one study [49] and the periods not indicated in the other study (follow-up varied for each participant) [8]. However, these articles rated poorly in their overall quality assessment, with "poor" to "fair" quality ratings of 3 [8] and 4 [49].

Smoking. Similar to nutrition, 4 (33\%) of the 12 genetic intervention studies had a positive influence on smoking-related behaviours. However, improvements in smoking-related behaviours were generally only sustained over a short-term period. The overall quality of these studies was "fair."

Sleep. It is clear that sleep is an understudied area of genetic testing and behaviour change research, since our comprehensive search did not yield a single study that assessed sleep (sleep quality, hours of sleep, etc.) as a behaviour change outcome.

Pooled Analyses. Two studies completed pooled analyses of changes in more than one lifestyle factor. Chao et al. [65] did not find significant changes in nutrition or physical activity on their own, but when pooled together, there were significantly greater changes to nutrition and physical activity in the high-risk genetic testing group than in the non-risk and control groups. Additionally, in a pooled analysis of changes to nutrition, physical activity, or smoking, Egglestone et al. [8] found significant changes between the genetic testing group and the control group. However, their results should be interpreted with caution, as this study was awarded the lowest overall quality rating of 3 (Table 3 ).

\section{Results from Controlled Intervention Trials}

While it is important to be comprehensive and consider all studies conducted on the topic of interest regardless of the research methods chosen, controlled interventions should be further highlighted and reviewed separately from other study designs given that this is the highest possible level of evidence for the original research included in the present review.

In total, 15 controlled intervention trials have been conducted over the past two decades. Approximately half of these studies ( $n=7 ; 47 \%)$ found significant changes in nutrition and/or physical activity or in smoking at 1-3 time points included in the study. Consistent with the overall analysis, the controlled interventions found that nutrition was the most promising area of behaviour change, followed by smoking (short-term only).

The genetic interventions in the controlled intervention trials overall ranked "fair," demonstrating that in comparison to the result of the pooled analysis of all study designs, these studies provided their participants with higher-quality genetic interventions. This may help explain why $47 \%$ of the controlled intervention studies found significant changes in lifestyle habits resulting from the genetic intervention, compared to $36 \%$ of the studies using other study designs. The overall ranking of these studies was "fair," with a mean rating of 5.6 out of the highest possible score of 9. Risk of bias overall was 
Table 3. Frequencies of genes tested in genetic interventions and their reported associated health outcomes

\begin{tabular}{|c|c|c|}
\hline Gene & Frequency & $\begin{array}{l}\text { Health outcomes reported to be } \\
\text { associated with the gene }\end{array}$ \\
\hline AAT & 1 & Emphysema \\
\hline $\mathrm{ACE}$ & 1 & Salt sensitivity \\
\hline apoE & 3 & $\begin{array}{l}\text { Alzheimer disease } \\
\text { Cardiovascular disease }\end{array}$ \\
\hline BRCA1 & 3 & $\begin{array}{l}\text { Breast cancer } \\
\text { Ovarian cancer }\end{array}$ \\
\hline BRCA2 & 3 & $\begin{array}{l}\text { Breast cancer } \\
\text { Ovarian cancer }\end{array}$ \\
\hline CYP1A2 & 1 & Caffeine metabolism \\
\hline CYP2D6 & 1 & Lung cancer \\
\hline FADS1 & 1 & Omega-3 metabolism \\
\hline FTO & 3 & Overweight/obesity \\
\hline GSMT1 & 3 & $\begin{array}{l}\text { Lung cancer } \\
\text { Vitamin } C \text { utilization }\end{array}$ \\
\hline GSTT1 & 1 & Vitamin C utilization \\
\hline KCNJ11 & 1 & Type 2 diabetes \\
\hline $\mathrm{L}-m y c$ & 2 & $\begin{array}{l}\text { Lung cancer } \\
\text { Oesophageal cancer }\end{array}$ \\
\hline NOD2 & 1 & Crohn disease \\
\hline PPAR $\gamma$ & 1 & Type 2 diabetes \\
\hline TAS1R2 & 1 & Sweet taste preference \\
\hline TCF7L2 & 1 & Type 2 diabetes \\
\hline
\end{tabular}

Of the studies that reported the specific genes tested in the genetic intervention, single nucleotide polymorphisms in 16 unique genes were tested, with apoE, BRCA1/2, FTO, and GSTM1 having the highest frequencies of use in the genetic intervention.

"fair" and consideration of the TPB was rated to be "poor," which is consistent with the results of the analysis of all study designs combined.

\section{Discussion}

Given that decoding the entire human genome was the primary focus of genetic research until 2003 [1], it is not surprising to find that the majority of studies included in the present review were published after this time, with only 2 studies published before 2003. Since then, much greater focus has been placed on genetic testing behaviour change research pertaining to nutrition, physical activity, and smoking. However, several studies included in the present review (46\%) did not include a COI statement. Future research should ensure the inclusion of a COI statement given this concerning finding and given the increased emphasis in academia on the importance of considering COI in genetic testing and other research.

Improving one or more of the four lifestyle behaviours of interest to this review has been shown to have a beneficial effect on chronic disease management and general health and well-being [20-23]. The present review indicated that improvements to smoking habits were promising in the short-term. This finding was consistent with that of a previously published systematic review of the impact of genetic notification on smoking cessation [66].

While nutrition, physical activity, and smoking habits have been researched in multiple genetic intervention studies, sleep remains an understudied area of genetics and behaviour change. This is notable considering the substantial impact that sleep has on overall health and well-being. Current systematic reviews demonstrate a significant impact of sleep on cognition and emotion [67], glycaemic control [22], and overweight or obesity [23], to name a few. To our knowledge, little is known about the ability of sleep to modify gene-associated health risks. Thus, future research should seek to first determine gene-sleep interactions that may influence health outcomes using methodologies similar to those of nutrigenomics research, as opposed to a genome-wide association study approach. Upon determining ways in which sleep may mitigate genetics-associated health risks, future research should then seek to determine if genetic testing helps to motivate healthy sleep-related behaviours.

The considerable heterogeneity in studies (Tables 1,2) can be explained by a number of factors. Notably, the variation of statistical analyses between groups (i.e., genetic testing groups vs. control groups or high-risk genetic result groups vs. non-risk genetic result groups) would have impacted the findings and subsequent conclusions drawn. Consideration of theories in general to inform the study design was poor, and consideration of the TPB was absent, which further helps to explain the heterogeneity of findings, since several possible confounding factors were missed. Additionally, only 3 studies $[9,11,43]$ focused on nutrient metabolism. Therefore, a future focus is needed on genetic interventions related to nutrient metabolism and the subsequent disease risk through genetic testing of modifier genes (genetic risks that can be mitigated through specific lifestyle changes), rather than genetics-based disease risk estimates where there may be no known lifestyle modifications that can alter the genetic risk. It is possible that nutrition was the most promising lifestyle factor for promoting health behaviour change given that genetic testing of modifier genes typically leads to the provision of actionable recom- 
mendations (e.g., the recommendation to reduce sodium intake [11]).

It is important to note that our risk-of-bias results are consistent with the previously published literature $[3,6]$, providing validation for the NIH quality assessment process completed in the current review. Effect sizes were not included in this review due to heterogeneity of the genetic interventions and study designs of the included articles that would have introduced potential flaws in effect size calculations and any conclusions drawn from such calculations. For randomized controlled trials, effect sizes have recently been presented elsewhere [3], although these should be interpreted with caution due to the significant heterogeneity of treatments (genetic interventions), measurements of outcomes, and populations studied. To our knowledge, we have developed and utilized the first quality assessment tool for evaluating and rating genetic interventions. Future research should seek to utilize this novel tool and significant contribution to the literature to assess the quality of genetic interventions in both primary research and systematic reviews. Furthermore, the components of this tool can be used in future genetic testing behaviour change study design to improve the quality of genetic interventions provided to participants (online suppl. Table 1). Although the genetic intervention quality assessment was based on previously published robust research and critical commentaries [11, 31-38], assessing the quality of evidence supporting the genetic tests provided to participants was beyond the scope of the present review. This is an important area of future research and is a notable ethical concern of genetic testing.

This review provides the most comprehensive analysis of genetic testing behaviour change research completed to date. However, some limitations to the present review exist. While this review summarized whether the genetic information was delivered direct to consumer or through a healthcare provider (Table 3 ), the practice of each provider is inevitably distinct. Some may incorporate behaviour change theory into their practice in order to maximally promote health behaviour change, while others may simply provide an explanation of the genetic results. This limitation further highlights the complexity of genetic testing behaviour change research. Additionally, the TPB was chosen as the key theory of interest given that it is one of the most widely accepted and validated theories of behaviour change, with over 4,500 publications referencing this theory and several meta-analyses finding that the key components of the TPB can be used to predict behavioural intentions with mean multiple correlations ranging from 0.59 to $0.67[15,68-73]$. However, a num- ber of other theories have been validated and are frequently used in behaviour change research, such as the transtheoretical model [74].

By improving upon genetic testing behaviour change studies, we anticipate the development of an algorithm that can be used to inform effective genetic testing behaviour change interventions for individuals who might benefit from this more personalized approach to healthcare. Indeed the limitations of genetic testing and the possible risk of harm [75] should be considered prior to an individual's decision to undergo genetic testing, especially in situations where one may learn about their risk of developing a disease, where actionable strategies for mitigating the risk are currently unknown [75]. Given that behaviour change is complex and multifactorial and studies have yet to robustly incorporate validated theory and high-quality genetic interventions into their methods, we cannot conclude with a broad statement about the impact of genetic testing on behaviour change. However, it is clear that it is possible to facilitate behaviour change through the provision of high-quality genetic interventions. Incorporating behaviour change theory into future research is an important consideration to enhance our knowledge in this field. Specific recommendations for study design have recently been published elsewhere [18]. An interdisciplinary research team with expertise in genomics as well as behaviour change may be the optimal approach given the complexities of this field of study. Considerable future research is needed in this promising and exciting area of lifestyle behaviour change research.

\section{Conclusion}

The use of validated theory to inform a robust study design [18] and the provision of actionable, high-quality, genetics-based information and advice is recommended to test a behaviour change hypothesis in genetics research. Rather than using the traditional systematic review process of assessing solely risk of bias, we have demonstrated that factors beyond risk of bias influence research outcomes related to genetic testing and behaviour change. As more robust literature continues to be published, allowing for the determination of key components of genetic interventions that best facilitate behaviour change, lifestyle genomics behaviour change research has the potential to make a substantial impact on global health and well-being through the facilitation of personalized, health-promoting lifestyle behaviour change. 


\section{Acknowledgements}

The authors would like to acknowledge Katarzyna Rabenda for her contribution to title and abstract screening.

\section{Disclosure Statement}

The authors declare that they have no conflicts of interest.

\section{References}

1 National Institutes of Health: All about the Human Genome Project. 2015. https://www. genome.gov/10001772/all-about-the-human-genome-project-hgp/.

$\checkmark 2$ Gibney MJ, Walsh MC: The future direction of personalised nutrition: my diet, my phenotype, my genes. Proc Nutr Soc 2013;72:219225.

- 3 Hollands GJ, French DP, Griffin SJ, Prevost AT, Sutton S, King S, Marteau TM: The impact of communicating genetic risks of disease on risk-reducing health behaviour: systematic review with meta-analysis. BMJ 2016 352:i1102.

-4 O’Donovan CB, Walsh MC, Gibney MJ, Brennan L, Gibney ER: Knowing your genes: does this impact behaviour change? Proc Nutr Soc 2017;76:182-191.

5 French DP, Cameron E, Benton JS, Deaton C, Harvie M: Can communicating personalised disease risk promote healthy behaviour change? A systematic review of systematic reviews. Ann Behav Med 2017;51:718-729.

6 Li SX, Ye Z, Whelan K, Truby H: The effect of communicating the genetic risk of cardiometabolic disorders on motivation and actual engagement in preventative lifestyle modification and clinical outcome: a systematic review and meta-analysis of randomised controlled trials. Br J Nutr 2016;116:924-934.

7 Caulfield T, McGuire AL: Direct-to-consumer genetic testing: perceptions, problems, and policy responses. Annu Rev Med 2012;63: 23-33.

8 Egglestone C, Morris A, O’Brien A: Effect of direct-to-consumer genetic tests on health behaviour and anxiety: a survey of consumers and potential consumers. J Genet Couns 2013;22:565-575.

-9 Hietaranta-Luoma HL, Tahvonen R, IsoTouru T, Puolijoki H, Hopia A: An intervention study of individual, apoE genotype-based dietary and physical-activity advice: impact on health behavior. J Nutrigenet Nutrigenomics 2014;7:161-174.

10 Marsaux CFM, Celis-Morales C, Livingstone KM, Livingstone KM, Fallaize R, Kolossa S, Hallmann J, et al: Changes in physical activity following a genetic-based internet-delivered personalized intervention: randomized controlled trial (Food4Me). J Med Internet Res 2016;18:e30.

11 Nielsen DE, El-Sohemy A: Disclosure of genetic information and change in dietary intake: a randomized controlled trial. PLoS One 2014;9:e112665
12 Roth GA, Johnson C, Abajobir A, Abd-Allah F, Abera SF, Abyu G, et al: Global, regional, and national burden of cardiovascular diseases for 10 causes, 1990 to 2015. J Am Coll Cardiol 2017;70:1-25.

13 CDC: Chronic disease overview. 2016. https:// www.cdc.gov/chronicdisease/overview/.

14 Knowler W, Barrett-Connor E, Fowler S, Hamman RF, Lachin JM, Walker EA, et al: Reduction in the incidence of type 2 diabetes with lifestyle intervention or metformin. $\mathrm{N}$ Engl J Med 2002;346:393-403.

15 Ajzen I: The theory of planned behaviour: reactions and reflections. Psychol Health 2011; 26:1113-1127.

16 Ajzen I: The theory of planned behavior. Organ Behav Hum Decis Process 1991;50:179211.

17 Ajzen I: Theory of planned behavior. 2006 http://people.umass.edu/aizen/tpb.html. (accessed April 11, 2017).

18 Horne J, Madill J, Gilliland J: Incorporating the "Theory of Planned Behaviour" into personalized healthcare behaviour change research: a call to action. Pers Med 2017;14: 521-529.

19 West S, King V, Carey TS, Lohr KN, McKoy $\mathrm{N}$, Sutton SF, et al: Systems to rate the strength of scientific evidence. Evid Rep Technol Assess (Summ) 2002;47:1-11.

20 Dean E, Söderlund A: What is the role of lifestyle behaviour change associated with noncommunicable disease risk in managing musculoskeletal health conditions with special reference to chronic pain? BMC Musculoskelet Disord 2015;16:87.

21 Walker KZ, O’Dea K, Gomez M, Girgis S, Colagiuri R: Diet and exercise in the prevention of diabetes. J Hum Nutr Diet 2010;23:344352

22 Zhu B, Hershberger PE, Kapella MC, Fritschi C: The relationship between sleep disturbance and glycaemic control in adults with type 2 diabetes: an integrative review. J Clin Nurs 2017;26:4053-4064.

$23 \mathrm{Wu}$ Y, Zhai L, Zhang D: Sleep duration and obesity among adults: a meta-analysis of prospective studies. Sleep Med 2014;15:14561462.

24 Audrain J, Boyd NNR, Roth J, Main D, Caporaso NE, Lerman C: Genetic susceptibility testing in smoking-cessation treatment: oneyear outcomes of a randomized trial. Addict Behav 1997;22:741-751.
25 Petticrew M, Roberts H: Systematic Reviews in the Social Sciences: A Practical Guide. Wiley-Blackwell, 2006.

26 Moher D, Liberati A, Tetzlaff J, Altman DG PRISMA Group: Preferred reporting items for systematic reviews and meta-analyses: the PRISMA statement. PLoS Med 2009;6: e1000097.

27 Food4Me: Food4Me: scientific publications. http://food4me.org/scientific-publications (accessed April 11, 2017).

28 Higgins J, Green S (eds): Cochrane Handbook for Systematic Reviews of Interventions, version 5.1.0 [updated March 2011]. The Cochrane Collaboration, 2011. http://handbook. cochrane.org.

29 National Institutes of Health: Study quality assessment tools. 2014. https://www.nhlbi. nih.gov/health-pro/guidelines/in-develop/ cardiovascular-risk-reduction/tools.

30 Critical Appraisal Skills Programme: CASP Qualitative Research Checklist, 2017. https:// casp-uk.net/casp-tools-checklists/.

31 Fenech M: Genome health nutrigenomics and nutrigenetics - diagnosis and nutritional treatment of genome damage on an individual basis. Food Chem Toxicol 2008;46:13651370.

32 Katsanis S, Katsanis N: Molecular genetic testing and the future of clinical genomics. Nat Rev Genet 2015;14:415-426.

33 Witte K, Meyer G, Martell D: Effective Health Risk Messages: A Step-By-Step Guide. Sage, Thousand Oaks, 2001.

34 Hall S, Weinman J, Marteau TM: The motivating impact of informing women smokers of a link between smoking and cervical cancer: the role of coherence. Health Psychol 2004;23:419-424.

35 Legenthal H, Benyamini $\mathrm{Y}$, Brownlee S, et al: Illness representations: theoretical foundations; in Petrie K, Weinman J (eds): Perceptions of Health and Illness: Current Research and Applications. Amsterdam, Harwood, 1997, pp 19-45.

36 Bloss CS, Schork NJ, Topol EJ: Effect of direct-to-consumer genomewide profiling to assess disease risk. N Engl J Med 2011;364: 524-534.

37 Bloss CS, Wineinger NE, Darst BF, Schork NJ, Topol EJ: Impact of direct-to-consumer genomic testing at long term follow-up. J Med Genet 2013;50:393-400

38 Ferguson LR, Barnett MPG: Research in nutrigenomics and potential applications to practice. Nutr Diet 2012;69:198-202. 
39 Elo S, Kyngäs H: The qualitative content analysis process. J Adv Nurs 2008;62:107-115.

-40 Siscovick DS, Barringer TA, Fretts AM, Wu $\mathrm{JH}$, Lichtenstein $\mathrm{AH}$, Costello RB, et al: Omega-3 polyunsaturated fatty acid (fish oil) supplementation and the prevention of clinical cardiovascular disease: a science advisory from the American Heart Association. Circulation 2017;135:e867-e884.

41 Cornelis MC, El-Sohemy A, Kabagambe EK: Coffee, CYP1A2 genotype, and risk of myocardial infarction. JAMA 2006;295:11351141.

-42 Hietaranta-Luoma H-L, Tahvonen R, IsoTouru T, Puolijoki H, Hopia A: An intervention study of individual, apoE genotype-based dietary and physical-activity advice: impact on health behavior. J Nutrigenet Nutrigenomics 2014;7:161-174.

43 Roke K, Walton K, Klingel S, Harnett A, Subedi S, Haines J, Mutch DM: Evaluating changes in omega-3 fatty acid intake after receiving personal FADS1 genetic information: a randomized nutrigenetic intervention. $\mathrm{Nu}$ trients 2017;9:E240.

44 Meisel SF, Beeken RJ, van Jaarsveld CHM, Wardle J: Genetic susceptibility testing and readiness to control weight: results from a randomized controlled trial. Obesity (Silver Spring) 2015;23:305-312.

45 Boeldt DL, Schork NJ, Topol EJ, Bloss CS: Influence of individual differences in disease perception on consumer response to directto-consumer genomic testing. Clin Genet 2015;87:225-232.

-46 Voils CI, Coffman CJ, Grubber JM, Edelman D, Sadeghpour A, Maciejewski ML, et al: Does type 2 diabetes genetic testing and counseling reduce modifiable risk factors? A randomized controlled trial of veterans. J Gen Intern Med 2015;30:1591-1598.

-47 Marsaux CFM, Celis-Morales C, Fallaize R, Macready AL, Kolossa S, Woolhead C, et al: Effects of a web-based personalized intervention on physical activity in European adults: a randomized controlled trial. J Med Internet Res 2015;17:e231.

-48 Bloss CS, Wineinger NE, Darst BF, Schork NJ, Topol EJ: Impact of direct-to-consumer genomic testing at long term follow-up. J Med Genet 2013;50:393-400.

-49 Kaufman DJ, Bollinger JM, Dvoskin RL, Scott JA: Risky business: risk perception and the use of medical services among customers of DTC personal genetic testing. J Genet Couns 2012; 21:413-422.

50 Hollands GJ, Whitwell SCL, Parker RA, Prescott NJ, Forbes A, Sanderson J, et al: Effect of communicating DNA based risk assessments for Crohn's disease on smoking cessation: randomised controlled trial. BMJ 2012;345:e4708.
51 Vernarelli JA, Roberts JS, Hiraki S, Chen CA, Cupples LA, Green RC: Effect of Alzheimer disease genetic risk disclosure on dietary supplement use. Am J Clin Nutr 2010;91:14021407.

52 Hishida A, Terazawa T, Mamiya T, Ito H, Matsuo K, Tajima K, et al: Efficacy of genotype notification to Japanese smokers on smoking cessation - an intervention study at workplace. Cancer Epidemiol 2010;34:96100.

53 Quach J, Porter K, Leventhal H, Kelly KM: Health behaviors among Ashkenazi Jewish individuals receiving counseling for BRCA1 and BRCA2 mutations. Fam Cancer 2009;8: 241-250.

54 O'Neill SC, Kaufman E, DeMarco T, Peshkin BN, McKenna K, Shelby R, et al: Changes in diet and physical activity following BRCA $1 / 2$ testing. J Psychosoc Oncol 2008;26:63-80.

55 Chao S, Roberts JS, Marteau TM, Silliman R, Cupples LA, Green RC: Health behavior changes after genetic risk assessment for Alzheimer disease: the REVEAL Study. Alzheimer Dis Assoc Disord 2008;22:94-97.

56 Sanderson SC, Humphries SE, Hubbart C, Hughes E, Jarvis MJ, Wardle J: Psychological and behavioural impact of genetic testing smokers for lung cancer risk: a phase II exploratory trial. J Health Psychol 2008; 13:481494.

57 Rees G, Gaff C, Young MA, Martin PR: Health beliefs and behaviors of women who have received genetic counseling for breast cancer. J Genet Couns 2007;16:457-468.

58 Rief W, Conradt M, Dierk JM, Rauh E, Schlumberger P, Hinney A, et al: Is information on genetic determinants of obesity helpful or harmful for obese people? - A randomized clinical trial. J Gen Intern Med 2007;22: 1553-1559.

59 Carpenter M, Strange C, Jones Y, Dickson MR, Carter C, Moseley MA, et al: Does genetic testing result in behavioral health change? Changes in smoking behavior following testing for alpha-1 antitrypsin deficiency. Ann Behav Med 2007;33:22-28.

60 Ito H, Matsuo K, Wakai K, Saito T, Kumimoto $\mathrm{H}$, Okuma $\mathrm{K}$, et al: An intervention study of smoking cessation with feedback on genetic cancer susceptibility in Japan. Prev Med 2006;42:102-108.

61 Marteau T, Senior V, Humphries SE, Bobrow M, Cranston T, Crook MA, et al: Psychological impact of genetic testing for familial hypercholesterolemia within a previously aware population: a randomized controlled trial. Am J Med Genet A 2004;128A:285-293.
62 McBride CM, Bepler G, Lipkus IM, Lyna P, Samsa G, Albright J, et al: Incorporating genetic susceptibility feedback into a smoking cessation program for African-American smokers with low income. Cancer Epidemiol Biomarkers Prev 2002;11:521-528.

63 Evans D: Hierarchy of evidence: a framework for ranking evidence evaluating healthcare interventions. J Clin Nurs 2003;12:77-84.

64 Public Health Agency of Canada: Social determinants of health. 2016. http://cbpp-pcpe. phac-aspc.gc.ca/public-health-topics/socialdeterminants-of-health/ (accessed April 11, 2017).

65 Chao S, Roberts JS, Marteau TM, Silliman R, Cupples LA, Green RC: Health behavior changes after genetic risk assessment for Alzheimer disease: the REVEAL Study. Alzheimer Dis Assoc Disord 2008;22:94-97.

66 de Viron S, Van der Heyden J, Ambrosino E, Arbyn M, Brand A, Van Oyen H: Impact of genetic notification on smoking cessation: systematic review and pooled-analysis. PLoS One 2012;7:e40230.

67 Krause AJ, Simon EB, Mander BA, Greer SM, Saletin JM, Goldstein-Piekarski AN, et al: The sleep-deprived human brain. Nat Rev Neurosci 2017;18:404-418.

68 Notani AS: Moderators of perceived behavioral control's predictiveness in the theory of planned behavior: a meta-analysis. J Consum Psychol 1998;7:247-271.

69 Rivis A, Sheeran P: Descriptive norms as an additional predictor in the theory of planned behaviour: a meta-analysis. Curr Psychol 2003;22:218-223.

70 Wittmann W: A meta-analysis of the theory of reasoned action and the theory of planned behavior: the principle of compatibility and multidimensionality of beliefs as moderators; in Schulze R, Holling H, Bohning D (eds): Meta-Analysis: New Developments and Applications in Medical and Social Sciences. Seattle, Hogrefe and Huber, 2003, pp 219-250.

71 Armitage CJ, Conner M: Efficacy of the Theory of Planned Behaviour: a meta-analytic review. Br J Soc Psychol 2001;40(pt 4):471-499.

72 Cheung SF, Chan D: The role of perceived behavioral control in predicting human behavior: a meta-analytic review of studies on the Theory of Planned Behavior. Unpublished manuscript, 2000.

73 McEachan R, Taylor N, Harrison R, Lawton $\mathrm{R}$, Gardner P, Conner M: Meta-analysis of the reasoned action approach (RAA) to understanding health behaviors. Ann Behav Med 2016;50:592-612.

74 Prochaska JO, Velicer WF: The transtheoretical model of health behavior change. Am J Health Promot 1997;12:38-48.

75 National Institutes of Health: Genetics home reference. What are the risks and limitations of genetic testing? 2018. https://ghr.nlm.nih. gov/primer/testing/riskslimitations.
Genetic Testing and Lifestyle Behaviour Change
Lifestyle Genomics 2018;11:49-63 DOI: $10.1159 / 000488086$ 